\title{
Synthesis, Characterization and Anti Microbial Activity of Some Novel Heterocyclic Compounds Having Sulphamido Moiety
}

\author{
RAMBABU NUNNA ${ }^{2}$, D. RAMACHANDRAN ${ }^{2 *}$, \\ VIRAL B. MODI ${ }^{1}$ and KIRTI J.GOSWAMI ${ }^{1}$
}

${ }^{1}$ Department of Chemistry, Shri U. P. Arts, Smt. M. G. Panchal Science \& Shri V. L. Shah Commerce College, Pilvai, India

${ }^{2}$ Acharya Nagarjuna University, P. G. Centre, Nuzvid, Andhrapradesh, India

viralkumarmodi@gmail.com

Received 2 February 2013 / Accepted 18 March 2013

\begin{abstract}
Amino- $N$-[4-(4-chloro-phenyl)-6-(3,4-dimethyl-phenyl)-pyrimidin-2-yl]-benzensulfonamide (2) was prepared by the hydrolysis of $N$-\{4-[4-Chloro-phenyl\}-6-(3,4-dimethyl-phenyl)-pyrimidin-2ylsulfamoyl]-phenyl\}-acetamide (1). It was on-facile condensation reaction with various aromatic aldehydes yields Schiff bases /anils/azomethines (3a-h). These anils on reaction with maleic anhydride and succinic anhydride yield $2 \mathrm{H}$-pyrrole-2-ones (4a-h) and 2-pyrrolidinones (5a-h) respectively. The newly synthesized compounds were evaluated for their antibacterial and antifungal activities.
\end{abstract}

Keywords: 2H-Pyrrole-2-ones, 2-Pyrrolidinones, Facile condensation, Schiff base, Antimicrobial activity

\section{Introduction}

The development of sulphonamides is one of the most fascinating and informative fields in medicinal chemistry, highlighting the roles of skillful planning and serendipity in drug research. The discovery of sulphonamides marked the beginning of the chemotherapeutic area by making possible a direct attack on microbial infections ${ }^{1}$. Sulphonamides antibacterials continued to be used because they are effective, inexpensive and free of super infection problems of the broad-spectrum antibiotic ${ }^{2}$. As a part of surge of interest in heterocyclic that have been explored for developing pharmaceutically important molecule $2 \mathrm{H}$-pyrrole-2-ones ${ }^{3,4}$ and 2-pyrrolidinones ${ }^{5-7}$ have played an important role in medicinal chemistry. Moreover, they have been studied extensively because of their ready accessibility, diverse chemical reactivity, and broad spectrum of biological activities.

Pyrimidine derivatives occupy a unique position as leiodynamic agents, both as essential components of nucleic acids and also as therapeutic agents ${ }^{8,9}$. During the past years considerable evidence has been accumulated to demonstrate the efficiency of substituted $2 \mathrm{H}$-pyrrole-2-ones, 2-pyrrolidinones and sulphonamides ${ }^{10-14}$. 
Keeping in view of biological importance of this group, we replace them by pyrimidine moiety at N1-position of sulphanilamide and $2 \mathrm{H}$-pyrrole-2-ones/2-pyrrolidinones at N4-position in sulphanilamide and our approach clearly shows the biological importance of the coupled products. The research work is scanned in scheme 1.

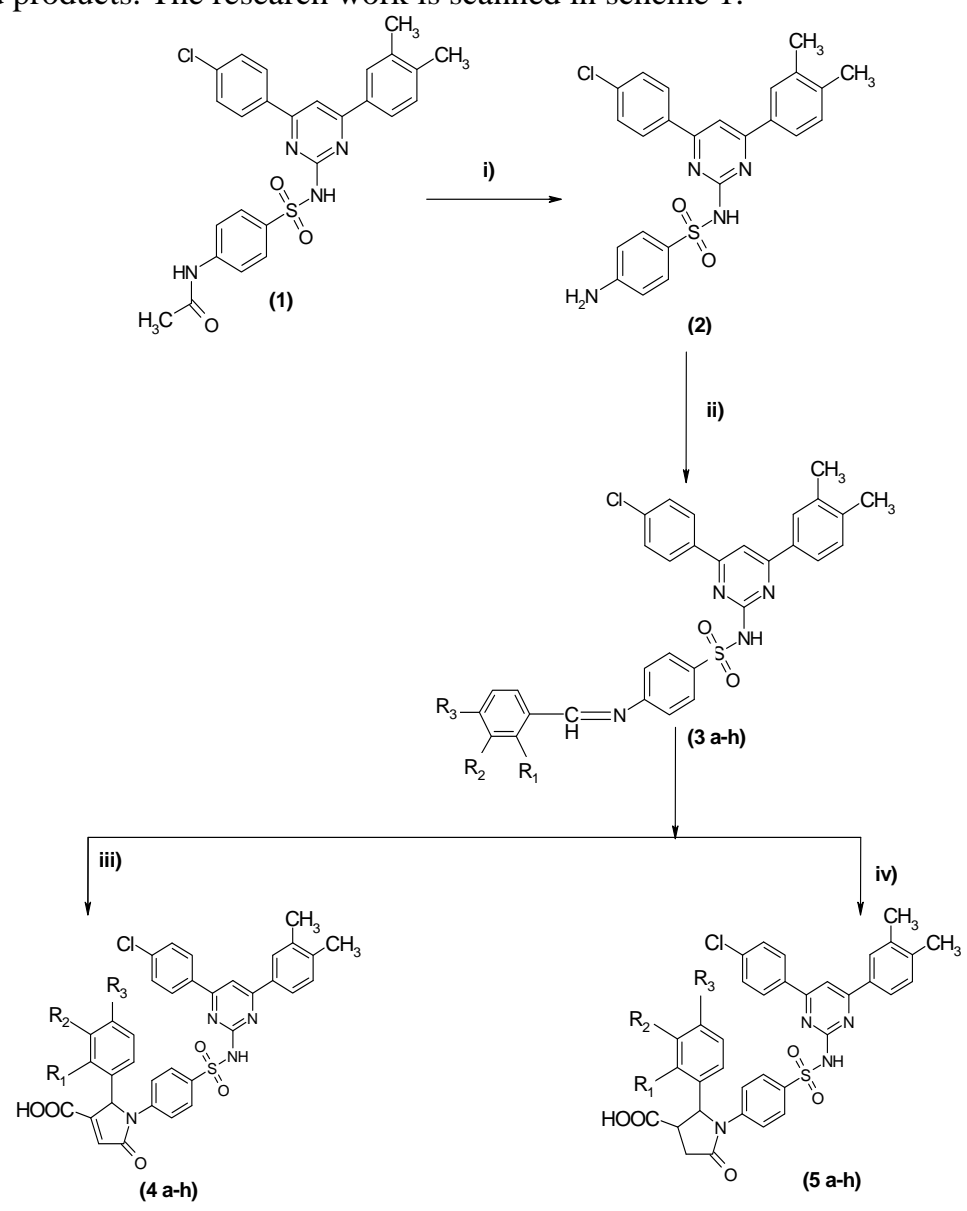

Scheme 1. Reagents and conditions: i) Hydrolysis/NaOH; ii) Ethanol/Substituted benzaldehyde / 8 h; iii) Maleic anhydride; iv) Succinic anhydride

(a) $\mathrm{R}_{1}=\mathrm{R}_{2}=\mathrm{R}_{3}=\mathrm{H}$; (b) $\mathrm{R}_{1}=\mathrm{R}_{2}=\mathrm{H}, \mathrm{R}_{3}=\mathrm{OCH}_{3}$; (c) $\mathrm{R}_{1}=\mathrm{R}_{2}=\mathrm{H}, \mathrm{R}_{3}=\mathrm{OH}$; (d) $\mathrm{R}_{1}=\mathrm{OH}, \mathrm{R}_{2}=\mathrm{R}_{3}=\mathrm{H}$;

(e) $\mathrm{R}_{1}=\mathrm{R}_{2}=\mathrm{H}, \mathrm{R}_{3}=\mathrm{CH}_{3}$; (f) $\mathrm{R}_{1}=\mathrm{H} \mathrm{R} \mathrm{R}_{2}=\mathrm{R}_{3}=-\mathrm{O}-\mathrm{CH}_{2}-\mathrm{O}-$; (g) $\mathrm{R}_{1}=\mathrm{H}, \mathrm{R}_{2}=\mathrm{OCH}_{3}, \mathrm{R}_{3}=\mathrm{OH}$;

(h) $\mathrm{R}_{1}=\mathrm{H}, \mathrm{R}_{2}=\mathrm{OCH}_{2} \mathrm{CH}_{3}, \mathrm{R}_{3}=\mathrm{OCH}_{2} \mathrm{CH}_{3}$

\section{Experimental}

Melting points were determined in open capillary tubes and are uncorrected. The IR spectra were recorded in $\mathrm{KBr}$ pellets on a Nicolet 400D spectrometer and ${ }^{1} \mathrm{H} \mathrm{NMR}$ spectra in $\mathrm{CDCl}_{3}$ on Hitachi R-1500, $60 \mathrm{MHz}$ spectrometer using TMS as an internal standard. The required $\mathrm{N}$-acetyl sulphanilyl chlorides (N-ASC) were prepared by reported method ${ }^{16}$. All chemicals used were of laboratory grade.

$N$-\{4-[4-Chloro-phenyl\}-6-(3,4-dimethyl-phenyl)-pyrimidin-2-ylsulfamoyl]-phenyl\}acetamide (1) was prepared according to the reported method ${ }^{17 a}$ 


\section{Antimicrobial activity}

\section{Antibacterial activity}

Antibacterial activities of all compounds were studied against gram positive (Bacillus subtillis and Staphylococcus aureus) and gram negative bacteria (E. coli and Salmonella typhi) at a concentration of $50 \mu \mathrm{g} / \mathrm{mL}$ by agar cup plate method ${ }^{15}$. Methanol system was used as control in this method. Under similar condition in penicilin and sulphamide as a standard comparison carried out controlled experiment. The area of inhibition of zone is measured in centimeters. Compounds $\mathbf{4 b}, \mathbf{4 c}, \mathbf{4 f}, \mathbf{5 b}$ and $\mathbf{5 f}$ were found more active against the above microbes. Other compounds found to be less or moderate active than the standards (Tables 1 and 2).

Table 1. Antibacterial activity and anti fungal activity of compounds (4a-h)

\begin{tabular}{ccccccc}
\hline & \multicolumn{4}{c}{ Antibacterial Activity } & \multicolumn{2}{c}{$\begin{array}{c}\text { Anti fungal } \\
\text { activity }\end{array}$} \\
\cline { 2 - 7 } Compounds & \multicolumn{4}{c}{ \% Zone of inhibition } \\
\cline { 2 - 7 } & \multicolumn{3}{c}{ Gram +ve } & \multicolumn{3}{c}{ Gram -ve } \\
B.Subtillis & S.Aureus & E.Coli & Ps.Aeruginosa & C. Albicans & A. Niger \\
\hline 4a & 47 & 40 & 46 & 61 & 43 & 41 \\
4c & 77 & 68 & 74 & 65 & 40 & 56 \\
4d & 56 & 45 & 40 & 54 & 51 & 45 \\
4e & 71 & 65 & 69 & 74 & 53 & 55 \\
$\mathbf{4 f}$ & 62 & 59 & 60 & 60 & 66 & 75 \\
$\mathbf{4 g}$ & 78 & 77 & 71 & 75 & 38 & 40 \\
4h & 57 & 58 & 55 & 48 & 63 & 71 \\
Penicillin & 49 & 39 & 59 & 60 & 45 & 68 \\
sulphanilamide & 83 & 67 & 77 & 74 & - & - \\
Griseofulvin & 79 & 72 & 83 & 70 & - & - \\
\hline
\end{tabular}

Table 2. Antibacterial activity and anti fungal activity of compounds (5a-h)

\begin{tabular}{|c|c|c|c|c|c|c|}
\hline \multirow{4}{*}{ Compounds } & \multicolumn{4}{|c|}{ Antibacterial Activity } & \multicolumn{2}{|c|}{$\begin{array}{l}\text { Anti fungal } \\
\text { activity }\end{array}$} \\
\hline & \multicolumn{6}{|c|}{ \% Zone of Inhibition } \\
\hline & \multicolumn{2}{|c|}{ Gram +ve } & \multicolumn{2}{|c|}{ Gram -ve } & \multirow[b]{2}{*}{ C. Albicans } & \multirow[b]{2}{*}{ A. Niger } \\
\hline & B.Subtillis & S.Aureus & E.Coli & Ps.Aeruginosa & & \\
\hline $5 a$ & 55 & 41 & 43 & 52 & 44 & 41 \\
\hline $5 \mathbf{b}$ & 60 & 58 & 64 & 56 & 66 & 76 \\
\hline $5 c$ & 50 & 47 & 58 & 48 & 51 & 44 \\
\hline 5d & 40 & 56 & 42 & 43 & 53 & 45 \\
\hline $5 e$ & 45 & 52 & 45 & 58 & 41 & 56 \\
\hline $5 f$ & 74 & 67 & 70 & 79 & 38 & 41 \\
\hline $5 g$ & 56 & 55 & 56 & 44 & 63 & 73 \\
\hline $5 \mathrm{~h}$ & 42 & 42 & 58 & 63 & 47 & 42 \\
\hline Penicillin & 84 & 66 & 77 & 75 & - & - \\
\hline sulphanilamide & 80 & 73 & 84 & 72 & - & - \\
\hline Griseofulvin & - & - & - & - & 79 & 83 \\
\hline
\end{tabular}


Antifungal activity

The compounds (4a-h) and (5a-h) were tested for in vitro antifungal activity against Candida. Albicans and Aspergillus Niger. The standard drug used was griseofulvin. The investigation antifungal screening is reported in Tables 1 and 2. Compounds $\mathbf{4 e ,} \mathbf{4 g}, \mathbf{5 b}$ and 5 g shows good activity against $C$. Albicans fungal strain.

Preparation of 4-amino- $N$-[4-(4-chloro-phenyl)-6-(3,4-dimethyl-phenyl)-pyrimidin-2-yl]benzensulfonamide (2) and 4-(arylidine-amino- $N$-[4-(4-chloro-phenyl)-6-(3,4-dimethyl-phenyl)pyrimidin-2-yl]-benzensulfonamide (3a-h) were made according to the reported method $^{17 \mathrm{~b}}$.

Preparation of 1-(4-( $\mathrm{N}$-(4-(4-chlorophenyl)-6-(3,4-dimethylphenyl) pyrimidin-2-yl)sul famoyl)phenyl)-5-oxo-2-aryl-2,5-dihydro-1H-pyrrole-3-carboxylic acid (4a-h) General procedure

A mixture of Schiff base (3a-h) $(0.1 \mathrm{~mol})$ and chloroform $\left(\mathrm{CHCl}_{3}\right)(40 \mathrm{~mL})$ was added with maleic anhydride $(0.1 \mathrm{~mol})$. Then it was refluxed for $8 \mathrm{~h}$ in an oil bath. After the mixture was allowed to stand for two days, the solid formed was filter then product was crystallized from absolute ethyl alcohol to give the compound (4a-h). Yield was 50-60\%.

1-(4-(N-(4-(4-Chlorophenyl)-6-(3,4-dimethylphenyl)pyrimidin-2-yl)sulfamoyl)phenyl) -5-oxo-2-phenyl-2,5-dihydro-1H-pyrrole-3-carboxylic acid (4a)

M.p 184-185 ${ }^{\circ} \mathrm{C}$; IR( $\left.\mathrm{KBr} \mathrm{cm}{ }^{-1}\right)$ : 3050(-Aromatic C-H-),1370,1150(-SO $\left.2^{-}\right), 1667(-\mathrm{COOH})$, 1717(-C=O); ${ }^{1} \mathrm{H}$ NMR:7.8 (s,1H,H-5- of the pyrimidne ring) ,6.12-7.56 (17H,m,Aromatic), 5.56 (s,1H, C2H), 11.02 (1H,s,-COOH); ${ }^{13} \mathrm{CMR}$ : 130(Benzene), 171.3(-COOH), 161.6 (-C=O); Anal Cald.for $\mathrm{C}_{35} \mathrm{H}_{27} \mathrm{~N}_{4} \mathrm{O}_{5} \mathrm{SCl}$ (651.13): C,64.56; H,4.18; N,8.60; S,4.92; Cl,5.44 Found: C,64.42; H,4.11; N,8.40; S,4.76; Cl,5.9; Yield 54\%;

1-(4-(N-(4-(4-Chlorophenyl)-6-(3,4-dimethylphenyl)pyrimidin-2-yl)sulfamoyl)phenyl) -2-(4-methoxyphenyl)-5-oxo-2,5-dihydro-1H-pyrrole-3-carboxylic acid (4b)

M.p 181-183 ${ }^{\circ} \mathrm{C}$; IR( $\left.\mathrm{KBr} \mathrm{cm}^{-1}\right)$ : 3050(-Aromatic C-H-),1370,1150(-SO $\left.2^{-}\right), 1667(-\mathrm{COOH})$, 1717(-C=O); ${ }^{1} \mathrm{H}$ NMR:7.8 (s,1H,H-5- of the pyrimidne ring ) ,6.12-7.56 (17H,m,Aromatic), $5.56\left(\mathrm{~s}, 1 \mathrm{H}, \mathrm{C}_{2} \mathrm{H}\right), 11.02(1 \mathrm{H}, \mathrm{s},-\mathrm{COOH}), 3.85\left(3 \mathrm{H}, \mathrm{s}, \mathrm{OCH}_{3}\right) ;{ }^{13} \mathrm{CMR}$ : 130(Benzene), 171.3 (-COOH), 161.6(-C=O), 56 (-OCH3); Anal Cald.for $\mathrm{C}_{36} \mathrm{H}_{29} \mathrm{~N}_{4} \mathrm{O}_{6} \mathrm{SCl}$ (681.16): C,63.48; H,4.29; N,8.23; S,4.71; Cl,5.20 Found: C,63.42; H,4.12; N,8.10; S,4.53; Cl,5.02; Yield 62\%; 1-(4-(N-(4-(4-Chlorophenyl)-6-(3,4-dimethylphenyl)pyrimidin-2-yl)sulfamoyl)phenyl) -2-(4-hydroxyphenyl)-5-oxo-2,5-dihydro-1H-pyrrole-3-carboxylic acid (4c)

M.p 188-189 ${ }^{\circ} \mathrm{C}$; IR(KBr cm$\left.{ }^{-1}\right)$ : 3050(-Aromatic C-H-),1370,1150(-SO $\left.2^{-}\right), 1667(-\mathrm{COOH})$, 1717(-C=O); ${ }^{1} \mathrm{H}$ NMR:7.8 (s,1H,H-5- of the pyrimidne ring), 6.12-7.56 (17H,m,Aromatic), 5.35 (s,1H,OH), 11.02 (1H,s,-COOH); ${ }^{13} \mathrm{CMR}$ : 130(Benzene), 171.3(-COOH), 161.6 $(-\mathrm{C}=\mathrm{O})$; Anal Cald.for $\mathrm{C}_{35} \mathrm{H}_{27} \mathrm{~N}_{4} \mathrm{O}_{6} \mathrm{SCl}$ (667.13): C,63.01; H,4.08; N,8.40; S,4.81; Cl,5.31 Found: C,63.00; H,3.89; N,8.27; S,4.81; Cl,5.31; Yield 56\%;

1-(4-(N-(4-(4-Chlorophenyl)-6-(3,4-dimethylphenyl)pyrimidin-2-yl)sulfamoyl)phenyl) -2-(4-hydroxyphenyl)-5-oxo-2,5-dihydro-1H-pyrrole-3-carboxylic acid (4d)

M.p 188-189 ${ }^{\circ} \mathrm{C}$; IR( $\left.\mathrm{KBr} \mathrm{cm}^{-1}\right)$ : 3050(-Aromatic C-H-),1370,1150(-SO $\left.{ }_{2}^{-}\right), 1667(-\mathrm{COOH})$, $1717(-\mathrm{C}=\mathrm{O})$; ${ }^{1} \mathrm{H}$ NMR:7.8 (s,1H,H-5- of the pyrimidne ring), 6.12-7.56 (17H,m,Aromatic), 5.35 (s,1H,OH), 11.02 (1H,s,-COOH); ${ }^{13} \mathrm{CMR}$ : 130(Benzene), 171.3(-COOH), 161.6(-C=O); Anal Cald.for $\mathrm{C}_{35} \mathrm{H}_{27} \mathrm{~N}_{4} \mathrm{O}_{6} \mathrm{SCl}$ (667.13): C,63.01; H,4.08; N,8.40; S,4.81; Cl,5.31 Found: C,62.95; H,3.85; N,8.20; S,4.80; Cl,5.27; Yield 58\%; 
1-(4-(N-(4-(4-Chlorophenyl)-6-(3,4-dimethylphenyl)pyrimidin-2-yl)sulfamoyl)phenyl) -5-oxo-2-p-tolyl-2,5-dihydro-1H-pyrrole-3-carboxylic(4e)

M.p 184-185 ${ }^{\circ} \mathrm{C}$; IR( $\left.\mathrm{KBr} \mathrm{cm}^{-1}\right)$ : 3050(-Aromatic C-H-),1370,1150(-SO $\left.{ }_{2}^{-}\right), 1667(-\mathrm{COOH})$, 1717(-C=O); ${ }^{1} \mathrm{H}$ NMR:7.8 (s,1H,H-5- of the pyrimidne ring), 6.12-7.56 (17H,m,Aromatic), 2.34 (s,3H, CH3), 11.02 (1H,s,-COOH),; ${ }^{13} \mathrm{CMR}:$ 130(Benzene), 171.3(-COOH), 161.6 $(-\mathrm{C}=\mathrm{O}), 21.2\left(-\mathrm{CH}_{3}\right)$; Anal Cald.for $\mathrm{C}_{36} \mathrm{H}_{29} \mathrm{~N}_{4} \mathrm{O}_{5} \mathrm{SCl}$ (665.16): C,65.00; H,4.39; N,8.06; S,4.60; Cl,5.33 Found: C,64.80; H,4.29; N,8.40; S,4.53; Cl,5.13; Yield 61\%;

2-(Benzo[d][1,3]dioxol-5-yl)-1-(4-(N-(4-(4-chlorophenyl)-6-(3,4-dimethylphenyl) pyrimidin-2-yl)sulfamoyl)phenyl)-5-oxo-2,5-dihydro-1H-pyrrole-3-carboxylic acid (4f)

M.p 183-184 ${ }^{\circ} \mathrm{C}$; IR( $\left.\mathrm{KBr} \mathrm{cm}^{-1}\right)$ : 3050(-Aromatic C-H-),1370,1150(-SO $\left.2_{2}^{-}\right), 1667(-\mathrm{COOH})$, 1717(-C=O); ${ }^{1} \mathrm{H}$ NMR:7.8 (s,1H,H-5- of the pyrimidne ring), 6.12-7.56 (17H,m,Aromatic ), 6.10 (s,2H, -O-CH2-O-), 11.02 (1H,s,-COOH),; ${ }^{13} \mathrm{CMR}: 130$ (Benzene), 171.3(-COOH), 161.6(-C=O), 101.2 , (-O-CH2-O-); Anal Cald.for $\mathrm{C}_{36} \mathrm{H}_{27} \mathrm{~N}_{4} \mathrm{O}_{7} \mathrm{SCl}$ (695.14): C,62.20; H,.91; N,8.06; S,4.61; Cl,5.10 Found: C,61.98; H,3.82; N,8.01; S,4.53; Cl,5.00; Yield 56\%;

1-(4-(N-(4-(4-Chlorophenyl)-6-(3,4-dimethylphenyl)pyrimidin-2-yl)sulfamoyl)phenyl) -2-(4-hydroxy-3-methoxyphenyl)-5-oxo-2,5-dihydro-1H-pyrrole-3-carboxylic acid (4g) M.p 186-185 ${ }^{\circ} \mathrm{C}$; IR( $\left.\mathrm{KBr} \mathrm{cm}^{-1}\right)$ : 3050(-Aromatic C-H-),1370,1150(-SO $\left.2^{-}\right), 1667(-\mathrm{COOH})$, 1717(-C=O); ${ }^{1} \mathrm{H}$ NMR:7.8 (s,1H,H-5- of the pyrimidne ring), 6.12-7.56 (17H,m, Aromatic), 3.83 (s,3H, -OCH3), 11.02 (1H,s,-COOH),; ${ }^{13} \mathrm{CMR}$ : 130(Benzene), 171.3(-COOH), 161.6 $(-\mathrm{C}=\mathrm{O}), 56$,(-OCH3); Anal Cald.for $\mathrm{C}_{36} \mathrm{H}_{29} \mathrm{~N}_{4} \mathrm{O}_{7} \mathrm{SCl}$ (697.14): C,62.02; H,4.19; N,8.04; S,4.60; Cl,5.09 Found: C,61.92; H,4.12; N,8.00; S,4.51; Cl,4.96; Yield 55\%;

1-(4-(N-(4-(4-Chlorophenyl)-6-(3,4-dimethylphenyl)pyrimidin-2-yl)sulfamoyl)phenyl) -2-(3,4-diethoxyphenyl)-5-oxo-2,5-dihydro-1H-pyrrole-3-carboxylic acid (4h)

M.p 187-188 ${ }^{\circ} \mathrm{C}$; IR( $\left.\mathrm{KBr} \mathrm{cm}{ }^{-1}\right)$ : 3050(-Aromatic C-H-),1370,1150(-SO $\left.{ }_{2}^{-}\right), 1667(-\mathrm{COOH})$, 1717(-C=O); ${ }^{1} \mathrm{H}$ NMR:7.8 (s,1H,H-5- of the pyrimidne ring), 6.12-7.56 (17H,m,Aromatic), 4.12 (4H of -2CH2-), 11.02 (1H,s,-COOH),; ${ }^{13} \mathrm{CMR}$ : 130(Benzene), 171.3(-COOH), 161.6 (-C=O), 65 ,(-OCH2); Anal Cald.for $\mathrm{C}_{39} \mathrm{H}_{35} \mathrm{~N}_{4} \mathrm{O}_{7} \mathrm{SCl}$ (773.28.14): C,63.37; H,4.77; N,7.58; S,4.34; Cl,4.80 Found: C,63.12; H,4.52; N,7.40; S,4.24; Cl,4.76; Yield 56\%;

Preparation of 1-(4-( $\mathrm{N}$-(4-(4-chlorophenyl)-6-(3,4-dimethylphenyl) pyrimidin-2-yl)sul famoyl)phenyl)-5-oxo-2-arylpyrrolidine-3-carboxylic acid (5a-h)

General procedure

A mixture of Schiff base (3a-h) $(0.1 \mathrm{~mol})$ and chloroform $\left(\mathrm{CHCl}_{3}\right)(40 \mathrm{~mL})$ was added with succinic anhydride $(0.1 \mathrm{~mol})$. Then it was refluxed for $8 \mathrm{~h}$ in an oil bath. After the mixture was allowed to stand for two days, the solid formed was filter then product was crystallized from absolute ethyl alcohol to give (5a-h). Yield was 50-60\%.

1-(4-(N-(4-(4-Chlorophenyl)-6-(3,4-dimethylphenyl)pyrimidin-2-yl)sulfamoyl)phenyl) -5-oxo-2-phenylpyrrolidine-3-carboxylic acid (5a)

M.p 188-189 ${ }^{\circ} \mathrm{C}$; IR( $\left.\mathrm{KBr} \mathrm{cm}^{-1}\right)$ : 3050(-Aromatic C-H-),1370,1150(-SO $\left.{ }_{2}^{-}\right), 1667(-\mathrm{COOH})$, 1717(-C=O); ${ }^{1} \mathrm{H}$ NMR:7.8 (s,1H,H-5- of the pyrimidne ring) ,6.12-7.56 (17H,m,Aromatic), 5.0(d,1H,C2 of Pyrrolidine ring), 2.5(d,2H,C4 of Pyrrolidine ring), 3.4(q,1H,C3 of Pyrrolidine ring), 11.02 (1H,s,-COOH); ${ }^{13} \mathrm{CMR}$ : 130(Benzene), 178.3(-COOH), 174.9(-C=O); Anal Cald.for $\mathrm{C}_{35} \mathrm{H}_{29} \mathrm{~N}_{4} \mathrm{O}_{5} \mathrm{SCl}$ (653.15): C,64.36; H,4.48; N,8.58; S,4.91; Cl,5.43 Found: C,64.06; H,4.28; N,8.50; S,4.82; Cl,5.41; Yield 64\%. 
1-(4-(N-(4-(4-Chlorophenyl)-6-(3,4-dimethylphenyl)pyrimidin-2-yl)sulfamoyl)phenyl) -2-(4-methoxyphenyl)-5-oxopyrrolidine-3-carboxylic acid (5b)

M.p 185-186 ${ }^{\circ} \mathrm{C}$; $\mathrm{IR}\left(\mathrm{KBr} \mathrm{cm}^{-1}\right)$ : 3050(-Aromatic C-H-),1370,1150(-SO $\left.{ }_{2}^{-}\right), 1667(-\mathrm{COOH})$, 1717(-C=O); ${ }^{1} \mathrm{H}$ NMR:7.8 (s,1H,H-5- of the pyrimidne ring), 6.12-7.56 (17H,m,Aromatic), 5.0(d,1H,C2 of Pyrrolidine ring), 2.5(d,2H,C4 of Pyrrolidine ring), 3.83(s,3H,- $\left.\mathrm{OCH}_{3}\right), 11.02$ (1H,s,-COOH); ${ }^{13} \mathrm{CMR}$ : 130(Benzene), 178.3(-COOH), 174.9(-C=O), 55.8 (-OCH3); Anal Cald.for $\mathrm{C}_{36} \mathrm{H}_{31} \mathrm{~N}_{4} \mathrm{O}_{6} \mathrm{SCl}$ (683.15): C,63.29; H,4.57; N,8.20; S,4.69; Cl,5.19 Found: C,63.21; H,4.31; N,8.11; S,4.60; Cl,5.12; Yield 54\%;

1-(4-(N-(4-(4-Chlorophenyl)-6-(3,4-dimethylphenyl)pyrimidin-2-yl)sulfamoyl)phenyl) -2-(4-hydroxyphenyl)-5-oxopyrrolidine-3-carboxylic acid (5c)

M.p 190-191 ${ }^{\circ} \mathrm{C}$; $\mathrm{IR}\left(\mathrm{KBr} \mathrm{cm}^{-1}\right)$ : 3050(-Aromatic C-H-),1370,1150(-SO $\left.{ }_{2}^{-}\right), 1667(-\mathrm{COOH})$, 1717(-C=O); ${ }^{1} \mathrm{H}$ NMR:7.8 (s,1H,H-5- of the pyrimidne ring), 6.12-7.56 (17H,m,Aromatic ), 5.0(d,1H,C2 of Pyrrolidine ring), 2.5(d,2H,C4 of Pyrrolidine ring), 5.35(s,3H,-OH), 11.02 (1H,s,-COOH); ${ }^{13} \mathrm{CMR}$ : 130(Benzene), 178.3(-COOH), 174.9(-C=O); Anal Cald.for $\mathrm{C}_{35} \mathrm{H}_{29} \mathrm{~N}_{4}$ $\mathrm{O}_{6} \mathrm{SCl}$ (653.15): C,62.82; H,4.37; N,8.37; S,4.79; Cl,5.30 Found: C,62.80; H,4.28; N,8.30; $\mathrm{S}, 4.69$; $\mathrm{Cl}, 5.22$; Yield 58\%;

1-(4-(N-(4-(4-Chlorophenyl)-6-(3,4-dimethylphenyl)pyrimidin-2-yl)sulfamoyl)phenyl) -2-(2-hydroxyphenyl)-5-oxopyrrolidine-3-carboxylic acid (5d)

M.p 188-189 ${ }^{\circ} \mathrm{C}$; $\mathrm{IR}\left(\mathrm{KBr} \mathrm{cm}^{-1}\right)$ : 3050(-Aromatic C-H-),1370,1150(-SO $\left.{ }_{2}^{-}\right), 1667(-\mathrm{COOH})$, 1717(-C=O); ${ }^{1} \mathrm{H}$ NMR:7.8 (s,1H,H-5- of the pyrimidne ring), 6.12-7.56 (17H,m,Aromatic), 5.0(d,1H,C2 of Pyrrolidine ring), 2.5(d,2H,C4 of Pyrrolidine ring), 5.35(s,3H,-OH), 11.02 (1H,s,-COOH); ${ }^{13} \mathrm{CMR}$ : 130(Benzene), 178.3(-COOH), 174.9(-C=O); Anal Cald.for $\mathrm{C}_{35} \mathrm{H}_{29} \mathrm{~N}_{4}$ $\mathrm{O}_{6} \mathrm{SCl}$ (653.15): C,62.82; H,4.37; N,8.37; S,4.79; Cl,5.30 Found: C,62.78; H,4.25; N,8.20; $\mathrm{S}, 4.62$; $\mathrm{Cl}, 5.20$; Yield 55\%;

1-(4-(N-(4-(4-Chlorophenyl)-6-(3,4-dimethylphenyl)pyrimidin-2-yl)sulfamoyl)phenyl) -5-oxo-2-p-tolylpyrrolidine-3-carboxylic acid (5e)

M.p 185-186 ${ }^{\circ} \mathrm{C}$; $\mathrm{IR}\left(\mathrm{KBr} \mathrm{cm}^{-1}\right)$ : 3050(-Aromatic C-H-),1370,1150(-SO $\left.{ }_{2}^{-}\right), 1667(-\mathrm{COOH})$, 1717(-C=O); ${ }^{1} \mathrm{H}$ NMR:7.8 (s,1H,H-5- of the pyrimidne ring), 6.12-7.56 (17H,m,Aromatic ), 5.0(d,1H,C2 of Pyrrolidine ring), 2.5(d,2H,C4 of Pyrrolidine ring), 2.34(s,3H, $\left.\mathrm{CH}_{3}\right), 11.02$ (1H,s,-COOH); ${ }^{13} \mathrm{CMR}: 130$ (Benzene), 178.3(-COOH), 174.9(-C=O) $21.3\left(-\mathrm{CH}_{3}\right)$; Anal Cald.for $\mathrm{C}_{36} \mathrm{H}_{31} \mathrm{~N}_{4} \mathrm{O}_{5} \mathrm{SCl}$ (667.17): C,64.81; H,4.68; N,8.40; S,4.81; Cl,5.31 Found: C,64.72; H,4.68; N,8.34; S,4.80; Cl,5.1; Yield 59\%;

2-(Benzo[d][1,3]dioxol-5-yl)-1-(4-(N-(4-(4-chlorophenyl)-6-(3,4-dimethylphenyl) pyrimidin-2-yl)sulfamoyl)phenyl)-5-oxopyrrolidine-3-carboxylic acid (5f)

M.p 178-179 ${ }^{\circ} \mathrm{C}$; IR( $\mathrm{KBr} \mathrm{cm}^{-1}$ ): 3050(-Aromatic C-H-),1370,1150(-SO2-), 1667(-COOH), 1717(-C=O) 1200 (Ar-O-Alkyl); ${ }^{1} \mathrm{H}$ NMR:7.8 (s,1H,H-5- of the pyrimidne ring), 6.12-7.56 (17H,m,Aromatic), $5.0(\mathrm{~d}, 1 \mathrm{H}, \mathrm{C} 2$ of Pyrrolidine ring), $2.5(\mathrm{~d}, 2 \mathrm{H}, \mathrm{C} 4$ of Pyrrolidine ring), 6.07(s,2H,-O-CH $\left.{ }_{2}-\mathrm{O}\right), 11.02$ (1H,s,-COOH); ${ }^{13} \mathrm{CMR}$ : 130(Benzene), 178.3(-COOH), 174.9 (-C=O), 101.2 (-O-CH2-O-); Anal Cald.for $\mathrm{C}_{36} \mathrm{H}_{29} \mathrm{~N}_{4} \mathrm{O}_{7} \mathrm{SCl}$ (697.16): C,62.02; H,4.19; N,8.04; S,4.60; Cl,5.09 Found: C,61.89; H,4.07; N,8.00; S,4.48; Cl,5.01; Yield 60\%;

1-(4-(N-(4-(4-Chlorophenyl)-6-(3,4-dimethylphenyl)pyrimidin-2-yl)sulfamoyl)phenyl) -2-(4-hydroxy-3-methoxyphenyl)-5-oxopyrrolidine-3-carboxylic acid (5g)

M.p 187-188 ${ }^{\circ} \mathrm{C}$; $\mathrm{IR}\left(\mathrm{KBr} \mathrm{cm}^{-1}\right)$ : 3050(-Aromatic C-H-),1370,1150(-SO $\left.{ }_{2}^{-}\right), 1667(-\mathrm{COOH})$, 1717(-C=O); ${ }^{1} \mathrm{H}$ NMR:7.8 (s,1H,H-5- of the pyrimidne ring) ,6.12-7.56 (17H,m,Aromatic), 
5.0(d,1H,C2 of Pyrrolidine ring), 2.5(d,2H,C4 of Pyrrolidine ring), 3.4(q,1H,C3 of Pyrrolidine ring), 11.02 (1H,s,-COOH) $5.35(1 \mathrm{H}, \mathrm{s},-\mathrm{OH}), 3.83\left(3 \mathrm{H}, \mathrm{s},-\mathrm{OCH}_{3}\right) ;{ }^{13} \mathrm{CMR}: 130$ (Benzene), 178.3(-COOH), 174.9(-C=O) $56.1\left(-\mathrm{OCH}_{3}\right)$; Anal Cald.for $\mathrm{C}_{36} \mathrm{H}_{31} \mathrm{~N}_{4} \mathrm{O}_{7} \mathrm{SCl}$ (699.17): C,61.84; H,4.47; N,8.01; S,4.59; Cl,5.07 Found: C,61.71; H,4.27; N,7.96; S,4.48; $\mathrm{Cl}, 4.90$; Yield 58\%;

1-(4-(N-(4-(4-Chlorophenyl)-6-(3,4-dimethylphenyl)pyrimidin-2-yl)sulfamoyl)phenyl) -2-(3,4-diethoxyphenyl)-5-oxopyrrolidine-3-carboxylic acid (5h)

M.p 168-169 ${ }^{\circ} \mathrm{C}$; IR( $\left.\mathrm{KBr} \mathrm{cm}{ }^{-1}\right)$ : 3050(-Aromatic C-H-),1370,1150(-SO $\left.{ }^{-}\right), 1667(-\mathrm{COOH})$, 1717(-C=O) 1200 (Ar-O-Alkyl); ${ }^{1} \mathrm{H}$ NMR:7.8 (s,1H,H-5- of the pyrimidne ring), 6.12-7.56 (17H,m,Aromatic), $5.0(\mathrm{~d}, 1 \mathrm{H}, \mathrm{C} 2$ of Pyrrolidine ring), 2.5(d,2H,C4 of Pyrrolidine ring), 3.4(q,1H,C3 of Pyrrolidine ring), $11.02(1 \mathrm{H}, \mathrm{s},-\mathrm{COOH}) 4.10\left(4 \mathrm{H}, \mathrm{q},-2 \mathrm{CH}_{2}\right), 1.34(6 \mathrm{H}, \mathrm{t},-$ 2 $\left.\mathrm{CH}_{3}\right)$; ${ }^{13} \mathrm{CMR}$ : 130(Benzene), 178.3(-COOH), 174.9(-C=O) $64.9\left(-\mathrm{OCH}_{2}-\right)$; Anal Cald.for $\mathrm{C}_{39} \mathrm{H}_{37} \mathrm{~N}_{4} \mathrm{O}_{7} \mathrm{SCl}$ ( 741.15 ): C,63.19; H,5.03; N,7.56; S,4.33; Cl,4.78 Found: C,63.11; H,4.93; N,7.36; S,4.13; Cl,4.67; Yield 61\%;

\section{Results and Discussion}

Since the antibacterial effect of sulphanilamide has been attributed to the presence of a sulphonamide groups $\left(-\mathrm{SO}_{2} \mathrm{NH}_{2}-\right)$ and $\mathrm{NH}_{2}$ group in para position, it is of interest to study the effect of fixation of these groups to the pyrimidine moiety. This interest has also prompted us to extend this study to include the effect of the introduction of the well known antibacterial nucleus (2H-pyrrole-2-ones /2-Pyrrolidinones) instead of $\mathrm{NH}_{2}$ group into the sulfa-pyrimidine nucleus.

The starting material, $N$-\{4-[4-chloro-phenyl\}-6-(3,4-dimethyl-phenyl)-pyrimidin-2ylsulfamoyl]-phenyl $\}$-acetamide (1) was prepared by according to the reported method $^{18}$. It can be hydrolyzed to 4-amino- $N$-[4-(4-chloro-phenyl)-6-(3,4-dimethyl-phenyl)-pyrimidin-2yl]-benzensulfonamide (2) by sodium hydroxide solution. It was characterized by the elemental analysis, IR spectral studies and NMR spectral studies. The strong absorptions at 1370 and 1160 were due to the presences of sulphonyl group. The structure of 2 was established by spectroscopic evidence.

This hydrolyzed product 2 was dissolved in absolute ethanol and was reacted with aromatic aldehyde in the presence of piperidine to yield Schiff bases (3a-h) were then characterized by the elemental analysis, IR spectral studies, and NMR spectral studies. The IR spectra of Schiff bases show the prominent band at $1630 \mathrm{~cm}^{-1}$ for the azomethine group ${ }^{18}$. All the compounds show the NMR signals for different kinds of protons at their respective positions. It was characterized by the elemental analysis, IR spectral studies and NMR spectral studies.

These Schiff bases on reaction with maleic anhydride and succinic anhydride yield $2 \mathrm{H}$-pyrrole-2-ones (4a-h) and 2-pyrrolidinones (5a-h) respectively. The structures of these compounds have been confirmed by elemental analysis, IR spectral studies and NMR spectral studies. These compounds shows the band at $1717 \mathrm{~cm}^{-1}$ for cyclic $(\mathrm{C}=\mathrm{O}$ of Pyrrole ring) group ${ }^{18}$. All the compounds show the NMR signals for different kinds of protons at their respective positions.

The antibacterial activities of both the series (4a-h) and (5a-h) respectively, have been carried out against some strain of bacteria. The results show that the prepared compounds are toxic against the bacteria. The comparison of the antibacterial activity of these compounds with penicillin and sulphanilamide shows that these compounds have almost similar activity. 


\section{Conclusion}

The clubbing of sulfa pyrimidine and $2 H$-pyrrole-2-ones /2-pyrrolidinones has been done successfully into one molecule. Both the moieties have important applications in medicinal use. The prepared compounds may be act as good biological compounds.

\section{Acknowledgment}

We are thankful to the department of chemistry, Himchandracharya North Gujarat University, Patan, Gujarat (India) for providing the necessary facilities for the research work.

\section{References}

1. Shepherd R G, Sulfanilamides and Other p-Aminobenzoic Acid Antagonists, Medicinal Chemistry, Edited by A. Burger, Wiley Interscience, Toronto , 1969 , 1, 255.

2. Krupp M A and Chatton M J, Current Medical Diagnosis and Treatment (Large Medical Publications, California, 1980).

3. David W Emerson, Richrd L Titus and Marlorr D Jones, J Heterocycl Chem., 1998, 35(3), 611-617.

4. Kuzuaki Oda, Hisao Tsujita, Masayuki Sakai and Machida Minoru, Chem Pharm Bull., 1998, 46(10), 1522-1526.

5. Thamotharan S, Parthasarathi V, Malik R, Jindal D P, Piplani P and Anthony Linden, Acta Crystallographica, Sec C: Cryst Str Comm., 2003, C59(9), 0514-0515.

6. Guindon Y and Bancheqroun M, Tetrahedron Lett., 2001, 42(35), 6041-6044.

7. Berry Peter Clark, Cynthia Lynn Cwi, John Richard Harris, Ann Elizabeth Kingston and William Leonard Scott, US pat. 00 69,816, 23; November 2000; C. A., 2001, 134 4857t.

8. Mochida Pharmaceutical Co. Ltd, Japan Pat 81,127,383, 1981; Chem Abstr., 1982, 96, 85572.

9. Ingram V M, Biosynthesis of Macromolecues, $2^{\text {nd }}$ Edition (Benjamin Menlo Park), 1972, 212.

10. Dash B, Praharaj S and Mohapatra P K, J Indian Chem Soc., 1981, LVIII, 1184.

11. Patel H S and Mistry H J, Phosphorous, Sulfur and Silicon and Related Elements, 2004, 179(6), 1085-1093.

12. Aboel-Magd A, Abdel-Wahab, Khairy M Hassan and Samia R El-Ezbawy, Indian J Chem., 1979, 18B, 467.

13. Hassan K H M, El-Ezbawy S R and Abdel-Wahab A A, J Indian Chem Soc., 1979, LVI, 290.

14. Hassan K H M and Atta F M, Indian J Chem., 1978, 16B, 1073.

15. a) Barry A L, The Antimicrobial Susceptibility Test : Principle and Practices, $4^{\text {th }}$ Ed., Edited by IIIuslea and feger (Philadelphila, 180-193, 1976; b) Biol Abstr., 1977, 64, 25183.

16. Vogel A I, A Textbook of Practical Organic Chemistry, $5^{\text {th }}$ Ed., Pearson Education, Ltd., Singapore, 2004, 883.

17. (a) El-hashash M A, Mahmoud M R and Madboli S A, Indian J Chem., 1993, 32B, 449-452; (b) Kokila A Parmar, Dhaval J Solanki and Devang J Solanki, Der Pharma Chemica, 2010, 2(5) , 358-364.

18. Bellamy L J, The Infrared Spectra of Complex Molecule (John Wiley and Sons, New York, 1954). 